\title{
What determines focal depth?
}

Qiuyun Liu ${ }^{1, *}$, Lipeng Liao ${ }^{1}$, Chanyuk Lam ${ }^{1}$, Yuhan Lin ${ }^{1}$, Wanquan Liu ${ }^{2}$, Man Tang ${ }^{1}$

${ }^{1}$ School of Life Sciences, Sun Yat-Sen University, Guangzhou 510275, China.

${ }^{2}$ Earthquake Monitoring and Prediction Department, Emergency Management Bureau, Deyang 618000, Sichuan, China.

*Correspondence author. E-mail address: liuqiuyunsysu@163.com (Q. Liu) 


\begin{abstract}
The interior of the Earth has smaller linear velocity than the Earth surface, but larger inertia due to gravity. This generates longer period of decelerations or accelerations in the interior producing strain with vertical and horizontal components. Faster linear velocity results in larger strain. Focal depth is the compromise of these two factors. Slender potential energy produces focal depth with hundreds of kilometers deep.
\end{abstract}


Seismic activities are similar to weight-lifting. Slower decelerations of large masses or faster accelerations of small masses, driven by solar and lunar pulls, can build up strain over a long time period $(1-5)$.

The interior of the Earth has smaller linear velocity than the Earth surface, but larger inertia due to intense gravity. This generates longer duration of decelerations or accelerations in the interior creating strain with vertical and horizontal components. The faster the linear velocity, the larger the strain, in a way similar to a fast-travelling car or train. The outcome is the tradeoff of these two factors, giving rise to focal depth. The place at the focal depth possesses larger vertical (upward) component than that in places straight up or straight down. The sharper the potential energy, the faster the speeds of decelerations or accelerations, like the top of a slender high-rise swaying the most visibly. Much deeper into the interior of the Earth has slow decelerations generating strain, which accounts for the deep focus earthquakes seen mostly near archipelagos. Only at this depth, the interior with slender potential energy on the Earth surface can build up adequate inertia to allow slow decelerations for longer durations for the creation of strain, eventually triggering seismic activities.

Acknowledgement:

We thank Yan Shi for editing.

\section{References}

1. Liao L., et al. Differential inertia of lower and upper parts of a skyscraper drives swaying of a building. OSF Preprints. 2021. doi:10.31219/osf.io/ztvsk. https://doi.org/10.31219/osf.io/ztvsk

2. Yan S, et al. Volcanoes or earthquakes: Wrist wrestling. Science, 2019; http://science.sciencemag.org/content/358/6370/1520/tab-e-letters.

3. Yan S, et al. Earthquakes-an intricate trio dance of gravitation. Science 2018; http://science.sciencemag.org/content/354/6315/1027/tab-e-letters.

4. Ran H, et al. Vortex-induced vibration: The slower deceleration of the west bank leads to the shortening of the Humen Bridge in Guangdong, China. OSF Preprints. 2020. doi:10.31219/osf.io/3t4a6. https://osf.io/3t4a6

5. Lu X, et al. 2020. The revolution of the earth around the sun causes the vortex-induced vibration of south-north trending bridges and impacts seismic activities. OSF Preprints. 2020. https://doi.org/10.31219/osf.io/ycjhz 